\title{
Utilization of Antibody Allows Rapid Clearance of Nanoparticle Probes from Blood Without the Need of the Probe Modifications
}

Akira Makino $^{* 1,2}$, Hidehiko Okazawa ${ }^{1,2}$, and Yasushi Kiyono ${ }^{1,2}$

1) Biomedical Imaging Research Center, University of Fukui, 23-3 Matsuokashimoaizuki, Eiheiji-cho, Yoshida-gun, Fukui 910-1193, Japan

2) Life Science Innovation Center, University of Fukui

KEYWORDS: Polymer micelle, antibody, positron emission tomography, tumor imaging

* Correspondence to Akira Makino: amakino@u-fukui.ac.jp 


\section{Figure S1}

(a)

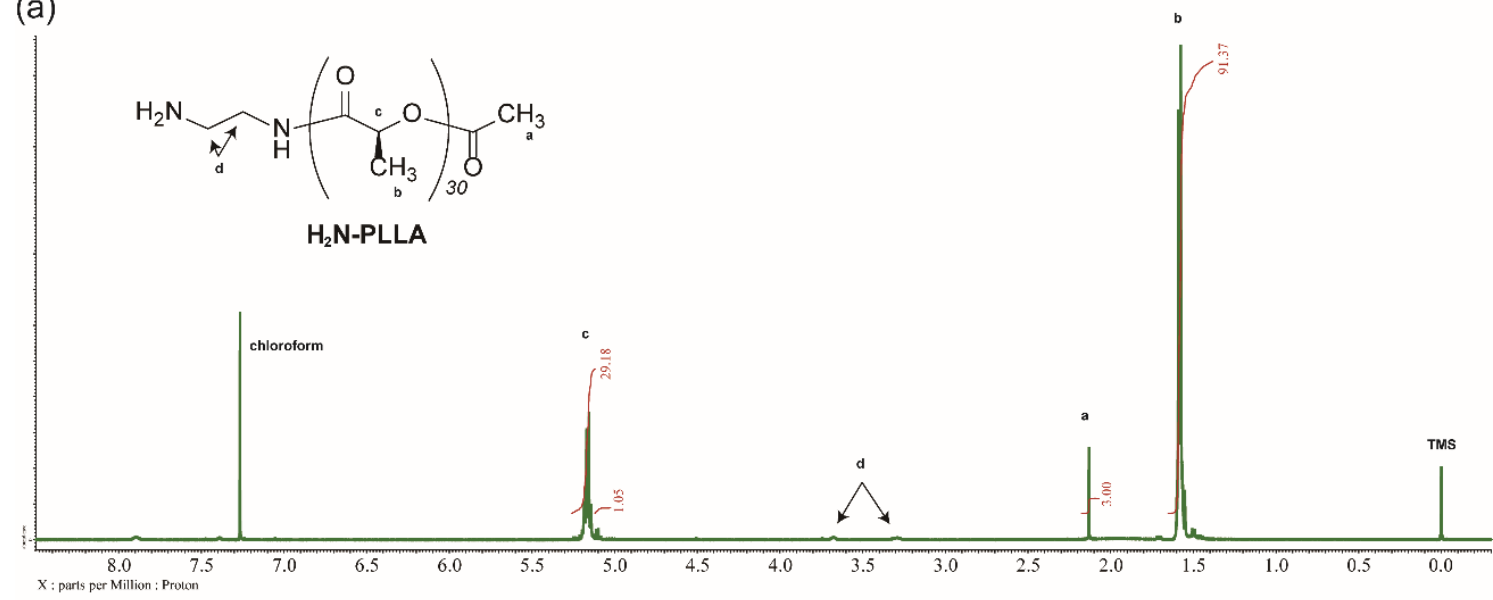

(b)

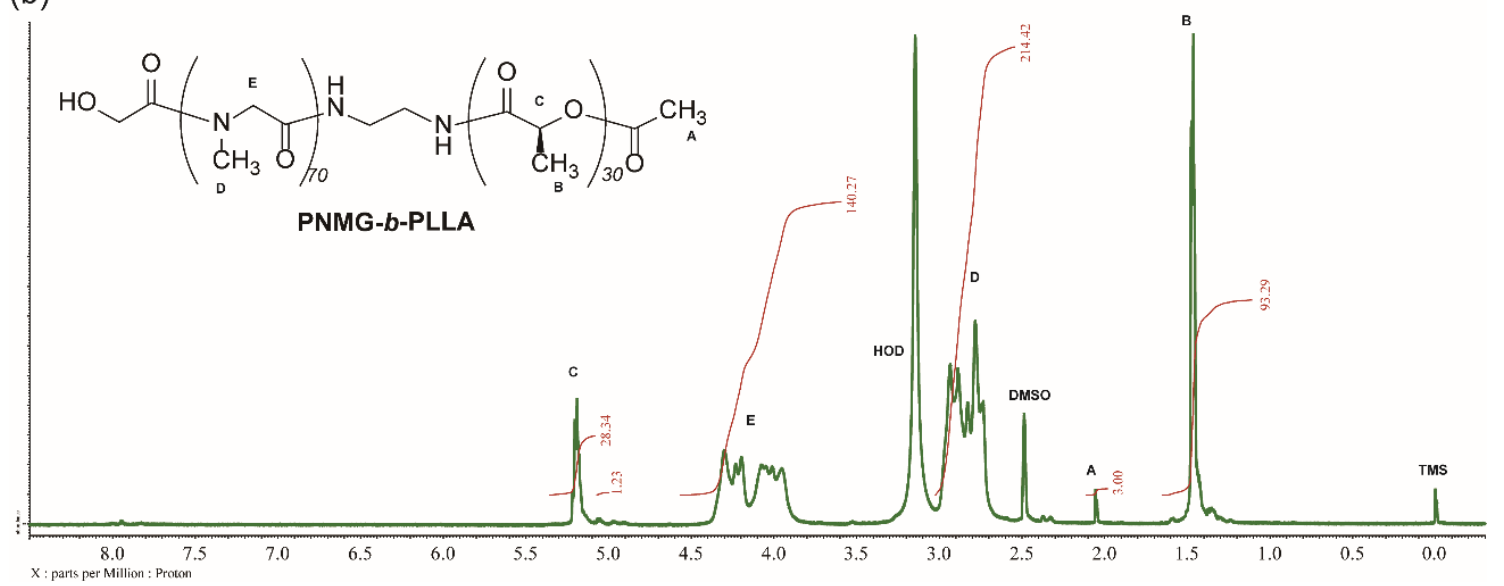

Figure S1. ${ }^{1} \mathrm{H}$ NMR spectra of (a) $\mathrm{H}_{2} \mathrm{~N}$-PLLA dissolved in chloroform- $d$ and (b) PNMG- $b$-PLLA in DMSO- $d_{6}$.

Polymer length was determined based on the integral value of acetyl group (2.1 ppm) located at the terminal end of PLLA unit. 


\section{Figure S2}

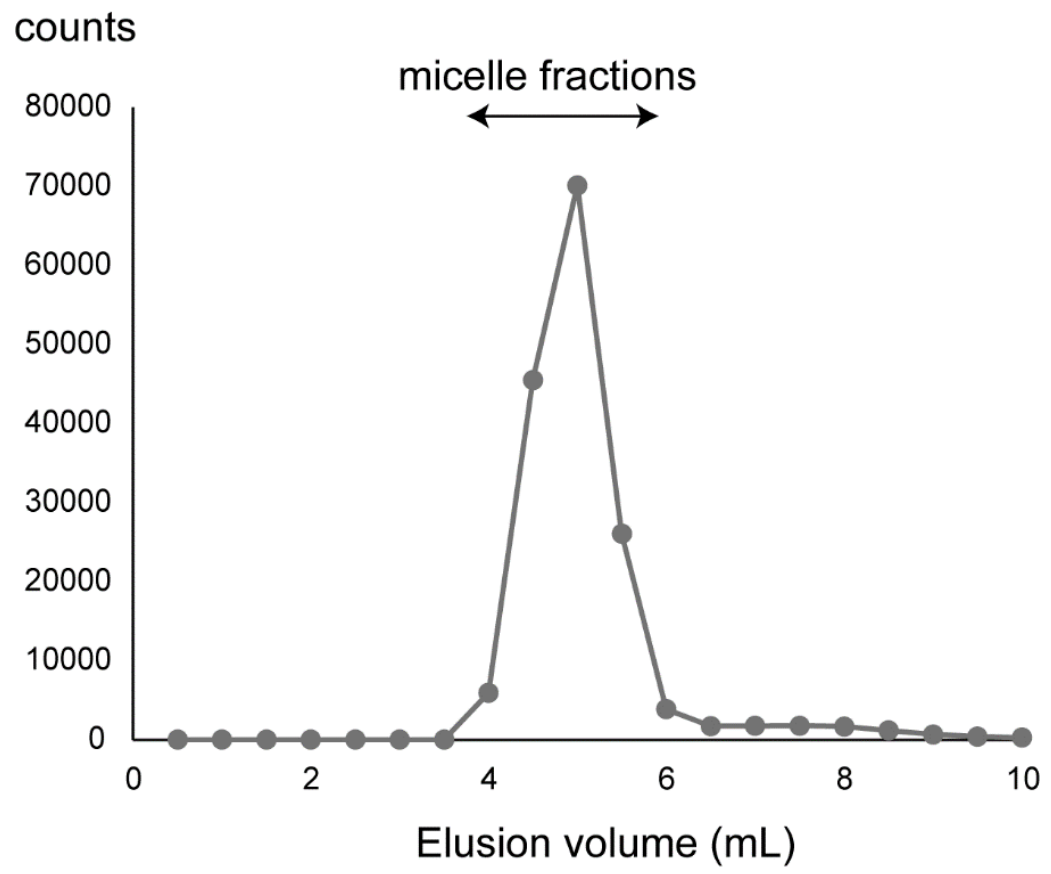

Figure S2. Elusion profile of ${ }^{18} \mathrm{~F}$-Lactosome using PD-10 column.

The micelle reaction mixture was passed through prepacked disposable PD-10 column (Cytiva, MA, USA) using PBS as an eluent. Figure S1 shows radioactivity profiles in the fractions collecting every $0.5 \mathrm{~mL}$. Radioactivity was measured using PerkinElmer Wallac Wizard 31480 automatic gamma counter (MA, USA). Only $\left[{ }^{18} \mathrm{~F}\right] \mathrm{FB}-\mathrm{PLLA}$ encapsulated into the micelle core was eluted from PD-10 because $\left[{ }^{18} \mathrm{~F}\right] \mathrm{FB}-\mathrm{PLLA}$ is hydrophobic and cannot dissolved into aqueous solutions. For the following experiments, mixture of 5 fractions from $4-6 \mathrm{~mL}$ was used. Encapsulation efficiency of ${ }^{18} \mathrm{~F}$-PLLA was calculated by the sum of radioactivity in fractions $(4-6 \mathrm{~mL})$ was divided by that bedded on the column, and determined to be $97.7 \%$. 
Figure S3

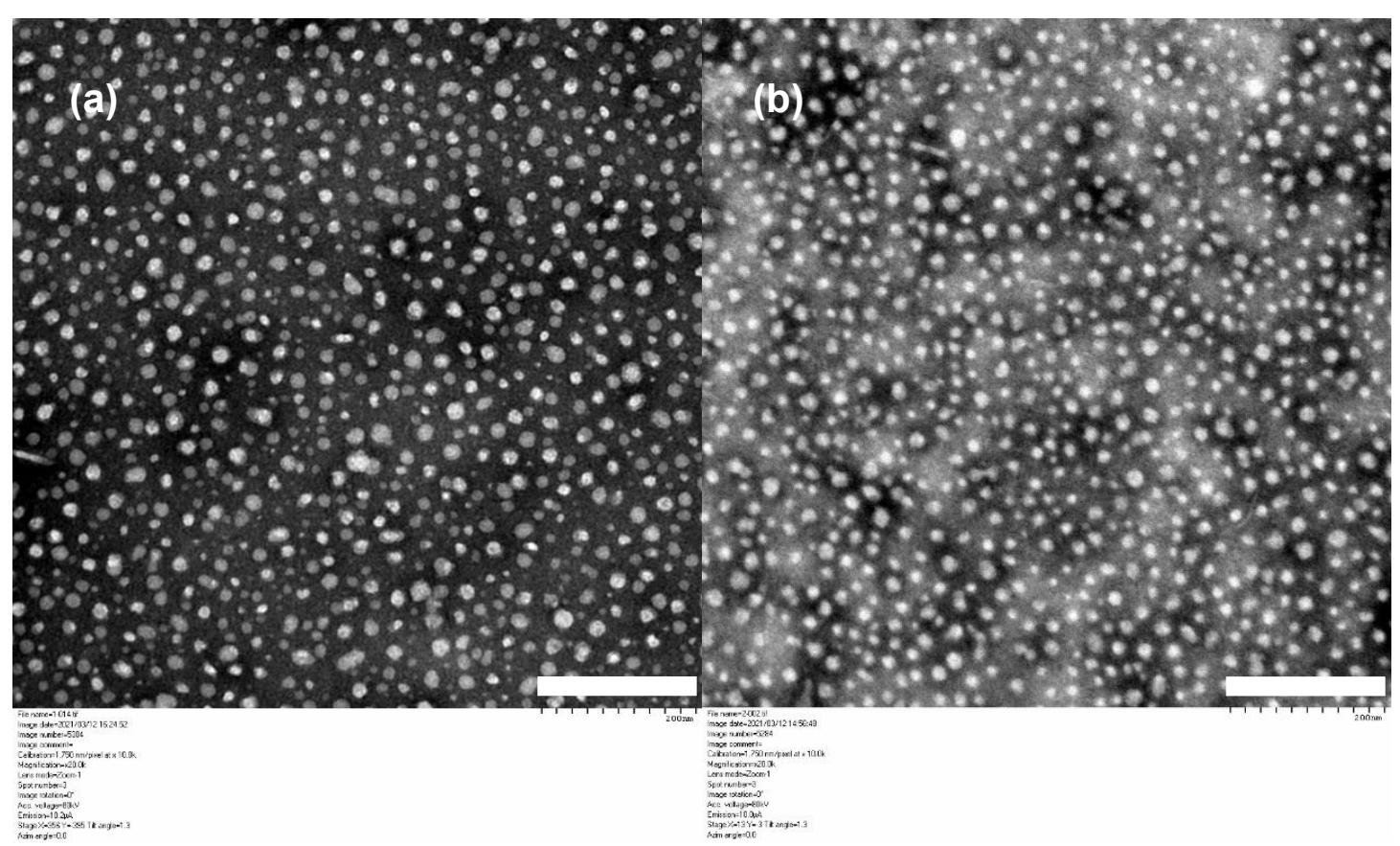

Figure S3. Transmission electron microscopic image of (a) Lactosome and (b) simulated ${ }^{18} \mathrm{~F}$-Lactosome. Scale bar: $200 \mathrm{~nm}$

Spherical micelle formation was evaluated using H-7650 transmission electron microscope at $80 \mathrm{keV}$ (Hitachi High-Tech, Japan). The sample was negatively stained by $1 \%$ phosphor tungstic acid. Due to the restrictions by Japanese law, radioactive compounds, including attenuated compounds, can only be used within controlled areas permitted by national agencies. At our institute, TEM is not installed in the controlled area, therefore, simulated ${ }^{18} \mathrm{~F}$-Lactosome was additionally prepared and used for TEM observation.

As explained in the manuscript, $\left[{ }^{18} \mathrm{~F}\right] \mathrm{FB}-\mathrm{PLLA}$ was synthesized by labeling reaction of primary amino group bearing PLLA 30 mer $1.5 \mathrm{mg}\left(\mathrm{H}_{2} \mathrm{~N}-\mathrm{PLLA}\right)$ using $\left[{ }^{18} \mathrm{~F}\right] \mathrm{SFB}$. The purification was performed by size exclusion chromatography, therefore, unreacted $\mathrm{H}_{2} \mathrm{~N}$-PLLA was left in the resulting $\left[{ }^{18} \mathrm{~F}\right] \mathrm{FB}-\mathrm{PLLA}$ $(1.5 \mathrm{mg})$. The important thing is radioisotopes are extremely sensitive, and molar amount of radiolabeled compound is very few. Indeed, $\left[{ }^{18} \mathrm{~F}\right] \mathrm{FB}$-PLLA $(1.5 \mathrm{mg})$ used for ${ }^{18} \mathrm{~F}$-Lactosome prearation is considered to be mostly $\mathrm{H}_{2} \mathrm{~N}$-PLLA on weight basis.

Then, simulated ${ }^{18} \mathrm{~F}$-Lactosome was prepared from the mixture of PNMG- $b$-PLLA $\left(18 \mathrm{mg}\right.$ ) and $\mathrm{H}_{2} \mathrm{~N}$ PLLA (1.5 mg). Both Lactosome (a) and simulated ${ }^{18} \mathrm{~F}$-Lactosome (b) forms core-shell type spherical micellar assembly. The core size is consistent with the value estimated from hydrodynamic diameter of the micelles determined by DLS measurements. 